\title{
The Methodical Approach to an Estimation of Camouflage Means Efficiency
}

Yuri L. Koziratsky, Andrey N. Smarov, Anton A. Koziratsky and Alexey V. Ivantsov* Military Education and Research Centre of Military-Air Forces «Military-Air Academy Named After Professor N.E. Zhukovsky and Yu.A. Gagarin» 54a Starykh Bol'shevikov Str., Voronezh, 394064, Russia

Received 11.09.2017, received in revised form 16.01.2018, accepted 27.02.2018

The methodical approach to an estimation of efficiency of the masking means is developed, allowing strictly enough to consider distributions of intensity of radiation on surfaces of object and a spreading surface. By embedding the amplitude distribution of the object radiation along the external surface of camouflaged means in the total distribution of the whole area received compound distribution in a subject plane with the further transfer of the image to a focal plane of sweeping system. It is shown, that at equal values of noise in images of the disguised object and a spreading surface distinction in signal levels in 5-10\% does not render essential influence on likelihood indicators of distinction.

Keywords: camouflage means, intensity of radiation, recognition.

Citation: Koziratsky Yu.L., Smarov A.N., Koziratsky A.A., Ivantsov A.V. The methodical approach to an estimation of camouflage means efficiency, J. Sib. Fed. Univ. Eng. technol., 2018, 11(3), 263-272. DOI: 10.17516/1999-494X-0038.

\section{Методический подход}

\section{к оценке эффективности маскировочных средств}

Ю.Л. Козирацкий, А.Н. Шмаров, А.А. Козирацкий, А.В. Иванцов Военный учебно-научный центр Военно-воздушных сил «Военно-воздушная академия имени профессора Н.Е. Жуковского и Ю.А. Гагарина» Россия, 394064, Воронеж, ул. Старых Большевиков, 54 а

Разработан методический подход $к$ оценке эффективности маскируюших средств, позволяющий достаточно строго учитывать распределения интенсивностей излучения

(C) Siberian Federal University. All rights reserved

* Corresponding author E-mail address: kagan13@yandex.ru 
по поверхностям объекта и подстилающей поверхности. Путем вложения амплитудного распределения излучения объекта по внешней поверхности маскирующего средства в суммарное распределение общей площади получено составное распределение в предметной плоскости с дальнейшим переносом изображения в фокальную плоскость системы разведки. Показано, что при равных значениях шумов в изображениях замаскированного объекта и подстилающей поверхности различие в уровнях сигнала в 5-10\% не оказывает существенного влияния на вероятностные показатели различения.

Ключевые слова: маскировочное средство, интенсивность излучения, распознавание.

Выбор и анализ средств маскировки объектов в ИК-диапазоне длин волн, когда в качестве разведывательного приемника используются приемники на основе матричных фотоэлементов, в настоящее время требуют достаточно строгих методических подходов [1-6]. С одной стороны, придание средствам маскировки возможности полного скрытия защищаемого объекта, когда его излучающий контраст на фоновом излучении подстилающей поверхности близок к нулю, существенно усложняют его использование в типовых боевых условиях. С другой стороны, пропускание средством маскировки всего лишь некоторой части излучения объекта и представление его в рассматриваемых типах ИК-систем разведки в виде изображения позволяют осуществлять достоверное обнаружение с использованием специальных алгоритмов распознавания (по фрагменту изображения объекта). Это подтверждает необходимость разработки строгого методического подхода к обоснованию способов и характеристик средства маскировки, которые обеспечили бы эффективное скрытие объекта и были бы пригодны к их широкому использованию без ограничений.

При такой постановке задачи различения в поле зрения разведывательного приемника, имеющего соответствующее площадное ограничение, будет попадать замаскированный объект и не прикрытая маскировочным средством (МC) часть подстилающей поверхности (ПП), что соответствует в нашей задаче гипотезе $H_{1}$. Когда в поле зрения наблюдается лишь ПП, ограниченная размерами поля зрения, то эта ситуация соответствует гипотезе $H_{0}$. В обоих случаях размеры площадей поверхностей излучающих объектов ограничены. В случае гипотезы $H_{1}$ общая ограниченная площадь поверхности, образованная полем зрения разведывательного приемника, разделяется на часть, которая прикрыта маскирующим средством (под которым, в свою очередь, находится объект и сугубо ПП), и на часть, которая представляет собой ПП. Для гипотезы $H_{0}$ должна рассматриваться общая ограниченная площадь поверхности, образованная полем зрения разведывательного приемника.

Наличие таких ограничений требует учета размеров излучающих и пропускающих площадей, что предполагает использование для описания образования и распространения излучения (изображения) метода «интегрирования по источнику». В этом случае с использованием функций размытия и начального амплитудного распределения излучения, исходящего с поверхности объекта, можно определить путем интегрирования по поверхности объекта амплитудное распределение излучения, исходящего от прикрываемого объекта, по внешней поверхности маскирующего средства. Это распределение «мозаичным образом» вкладывается в суммарное распределение общей площади, ограниченной полем зрения разведывательного ИК-приемника. Образованное таким путем составное распределение в предметной плоскости является в некотором смысле исходным для описания процесса пе- 


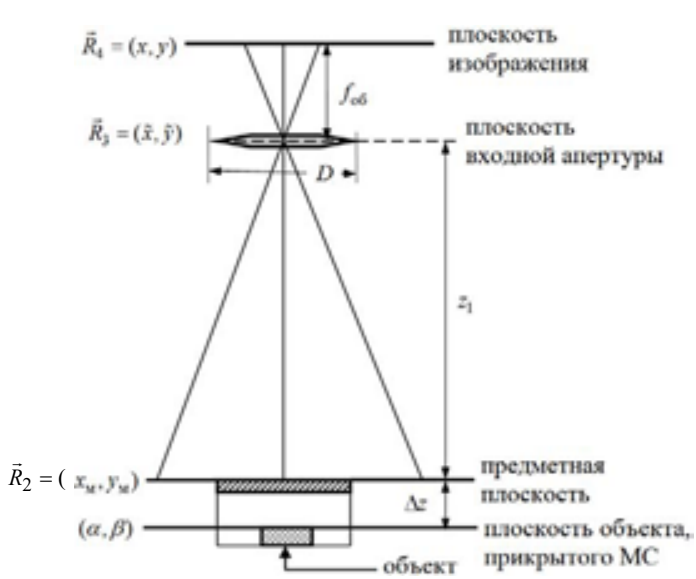

Рис. 1. Геометрия пассивной локации замаскированного объекта (ситуация, соответствующая гипотезе $H_{1}$ )

Fig. 1. Passive ranging geometry of camouflaged object (situation according to hypothesis $H_{1}$ )

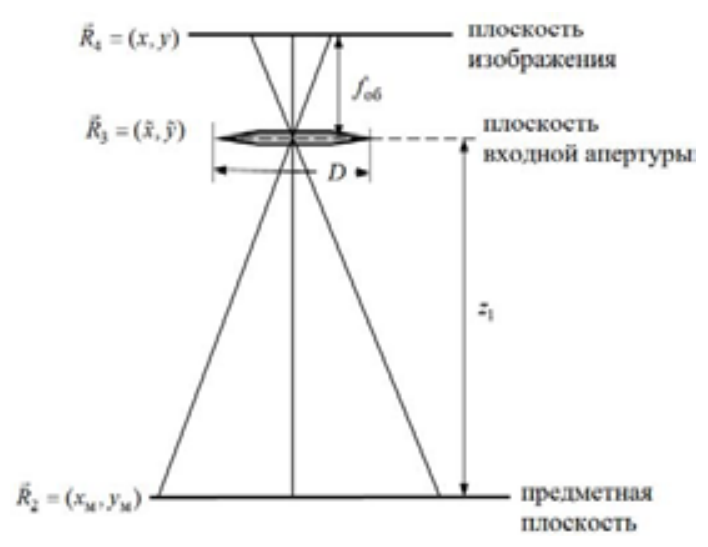

Рис. 2. Геометрия пассивной локации (ситуация, соответствующая гипотезе $H_{0}$ )

Fig. 2. Passive ranging geometry (situation according to hypothesis $H_{0}$ )

реноса изображения из рассматриваемой плоскости в фокальную (плоскость изображения) ИК-системы разведки.

С учетом изложенного рассмотрим задачу определения амплитудного распределения в плоскости изображения, соответствующего гипотезе $H_{1}$. На рис. 1 представлена геометрия пассивной локации замаскированного объекта с необходимыми обозначениями. Условия пассивной локации, соответствующие гипотезе $H_{0}$, представлены с необходимыми обозначениями на рис. 2.

Предположим, что амплитудное распределение излучения объекта задано и описывается функцией $U_{0}(\alpha, \beta)$. Амплитудную функцию размытия маскировочного средства обозначим через $F_{1}\left(x_{\mathrm{m}}, y_{\mathrm{м}}, \alpha, \beta\right)$. Тогда амплитудное распределение на внешней поверхности маскировочного средства будет иметь вид

$$
U_{2}^{\prime}\left(x_{\mathrm{M}}, y_{\mathrm{M}}\right)=\iint_{S_{\mathrm{o \sigma}}} U_{0}(\alpha, \beta) F_{1}\left(x_{\mathrm{M}}, y_{\mathrm{M}}, \alpha, \beta\right) d x d \beta=\iint_{S_{\mathrm{o \sigma}}} U_{0}\left(\vec{R}_{1}\right) F_{1}\left(\vec{R}_{2}, \vec{R}_{1}, \Delta z\right) d^{2} R_{1} .
$$

Интегрирование в данном случае ведется по поверхности объекта, подлежащего маскировке. Кроме того, будем полагать, что маскировочное средство обладает собственным излучением, так что амплитудное распределение на внешней поверхности маскировочного средства представляется в виде

$$
U_{2}\left(x_{\mathrm{M}}, y_{\mathrm{M}}\right)=U_{2}^{\prime}\left(x_{\mathrm{M}}, y_{\mathrm{M}}\right)+\Delta U_{2}\left(x_{\mathrm{M}}, y_{\mathrm{M}}\right),
$$

где $\Delta U_{2}\left(x_{\mathrm{m}}, y_{\mathrm{s}}\right)$ - амплитудное распределение на внешней поверхности маскировочного средства, вызванное собственным распределением.

Амплитудное распределение подстилающей поверхности может носить любой характер в зависимости от температурных и атмосферных условий, растительности, состава почвы, времени года. Обозначим амплитудное распределение подстилающей поверхности через

$$
-265-
$$


$U_{2 \text { пा }}\left(\bar{x}_{\mathrm{M}}, \bar{y}_{\mathrm{M}}\right)$, где координаты $\bar{x}_{\mathrm{M}}, \bar{y}_{\mathrm{M}}$ находятся вне внешней поверхности маскирующего средства. Следовательно, в предметной плоскости амплитудное распределение представляет собой сумму распределений, имеющей различную природу образования:

$$
\begin{aligned}
& U_{2 \Sigma}\left(x_{\mathrm{M}}, y_{\mathrm{M}}\right)=U_{2}^{\prime}\left(x_{\mathrm{M}}, y_{\mathrm{M}}\right)+\Delta U_{2}\left(x_{\mathrm{M}}, y_{\mathrm{M}}\right)+U_{2 \pi \Pi}\left(\bar{x}_{\mathrm{M}}, \bar{y}_{\mathrm{M}}\right)= \\
& =U_{2}\left(x_{\mathrm{M}}, y_{\mathrm{M}}\right)+U_{2 \pi \Pi}\left(\bar{x}_{\mathrm{M}}, \bar{y}_{\mathrm{M}}\right) .
\end{aligned}
$$

Амплитудное распределение для гипотезы $H_{1}$ в плоскости изображения находится (в случае невозмущенной среды распространения) на основе интегрирования по соответствующим источникам:

$$
\begin{aligned}
& U_{1}(x, y)=\iint_{S_{\mathrm{M}}} U_{2}\left(x_{\mathrm{M}}, y_{\mathrm{M}}\right) F_{2.4}\left(x, y, x_{\mathrm{M}}, y_{\mathrm{M}}\right) d x_{\mathrm{M}} d y_{\mathrm{M}}+ \\
& +\iint_{\Pi_{2}} U_{2 п \Pi}\left(\bar{x}_{\mathrm{M}}, \bar{y}_{\mathrm{M}}\right) F_{2.4}\left(x, y, \bar{x}_{\mathrm{M}}, \bar{y}_{\mathrm{M}}\right) d \bar{x}_{\mathrm{M}} d \bar{y}_{\mathrm{M}},
\end{aligned}
$$

в выражении (4)

$$
\begin{aligned}
& F_{2.4}\left(x, y, x_{\mathrm{M}}, y_{\mathrm{M}}\right)=\frac{1}{\bar{\lambda} z_{1}} e^{j \frac{\pi}{\bar{\lambda} z_{1}}\left(x_{\mathrm{M}}^{2}+y_{\mathrm{M}}^{2}\right)} \frac{1}{\bar{\lambda} f_{\mathrm{o \sigma}}} e^{j \frac{\pi}{\bar{\lambda} f_{\mathrm{o \sigma}}}\left(x^{2}+y^{2}\right)} \times \\
& \times \iint_{\infty} P_{\mathrm{c}}(\tilde{x}, \tilde{y}) e^{-j \frac{\pi}{f_{\mathrm{o} \sigma}}\left[\left(x+M x_{\mathrm{M}}\right) \tilde{x}+\left(y+M y_{\mathrm{M}}\right) \tilde{y}\right]} d \tilde{x} d \tilde{y},
\end{aligned}
$$

где $\tilde{x}, \tilde{y}$ - координаты во входной плоскости формирующей оптики (рис. 1 и 2 ); $M=f_{\text {об }} / z_{1}$; $\bar{\lambda}-$ средняя длина оптической волны; $P_{\mathrm{c}}(\tilde{x}, \tilde{y})$ - функция зрачка объектива.

В первом слагаемом интегрирование ведется по внешней поверхности маскировочного средства, а во втором - по поверхности подстилающей поверхности, исключая внешнюю поверхность маскировочного средства, и в пределах поверхности, ограниченной полем зрения средства ИК-разведки.

При известном амплитудном распределении $U(x, y)$ распределение интенсивности устанавливается путем усреднения произведения комплексно-сопряженных значений $U(x, y)[7]$

$$
I_{1}(x, y, t)=\left\langle U(x, y, t) \cdot U^{*}(x, y, t)\right\rangle .
$$

В этом случае амплитудное распределение в плоскости изображения

$$
U_{0}(x, y)=\iint_{S_{\Pi 3}} U_{2 \pi \Pi}\left(x_{\mathrm{M}}, y_{\mathrm{M}}\right) F_{2.4}\left(x, y, x_{\mathrm{M}}, y_{\mathrm{M}}\right) d x_{\mathrm{M}} d y_{\mathrm{M}} .
$$

Интегрирование выполняется по всей поверхности, ограниченной полем зрения разведывательного ИК-приемника.

Распределение интенсивности в плоскости изображения, обусловленной излучением подстилающей поверхности, находящейся в поле зрения разведывательного ИК-приемника, определим аналогично предыдущему случаю:

$$
\begin{array}{r}
I_{0}(x, y, t)=\left\langle U_{0}(x, y, t) \cdot U_{0}^{*}(x, y, t)\right\rangle . \\
-266-
\end{array}
$$


Если учесть фоновое излучение и внутренние шумы приемника (пересчитанные на вход в виде добавки к фоновому излучению), то можно каждой гипотезе, подлежащей различению, поставить в соответствие аддитивные смеси:

$$
\begin{aligned}
& H_{1}: \xi(x, y, t)=I_{1}(x, y, t)+n_{1}(x, y, t) ; \\
& H_{0}: \xi(x, y, t)=I_{0}(x, y, t)+n_{0}(x, y, t) .
\end{aligned}
$$

Определим «за противника» оптимальный алгоритм обработки входного воздействия, обеспечивающего наилучшим образом (в смысле минимального значения суммарной ошибки) определение, что в принимаемой смеси $\xi(x, y, t)$ содержится сигнал $I_{1}(x, y, t)$ или сигнал $I_{0}(x, y, t)$. При этом гипотезы составляют полную группу событий, так что сумма вероятностей $P\left(H_{1}\right)+P\left(H_{0}\right)=1$.

Размеры элемента матричного ИК-приемника удовлетворяют условию $\Delta S=\left(x_{i}-x_{i-1}\right) \cdot\left(y_{j}-y_{j-1}\right)=\Delta x \cdot \Delta y=\Delta_{1} \cdot \Delta_{1}=\Delta_{1}^{2}$, где $\Delta S$ - чувствительная площадь поверхности элемента приемника. Величину сигнала (тока), снимаемого с элемента, имеющего координаты $\left(x_{i}, y_{i}\right)$, можно определить с помощью выражения

$$
\xi(i, j)=\frac{\eta e}{h \bar{v}} \int_{x_{i}-\Delta_{1}}^{x_{i}} \int_{y_{j}-\Delta_{1}}^{y_{j}} \xi(x, y) d x d y,
$$

где $e$ - заряд электрона; $h$ - постоянная Планка; $\bar{v}$ - средняя частота оптических колебаний.

Применительно к сигналу от замаскированного объекта можем записать:

$$
\dot{I}_{1}(i, j)=\frac{\eta e}{h \bar{v}} \int_{x_{i}-\Delta_{1}}^{x_{i}} \int_{y_{j}-\Delta_{1}}^{y_{j}} S_{1}(x, y, t) d x d y .
$$

Шум, обусловленный фоновым излучением и, возможно, излучением постановщика помех, прикрывающего объект, описывается выражением

$$
n_{1}(i, j)=\frac{\eta e}{h \bar{v}} \int_{x_{i}-\Delta_{1}}^{x_{i}} \int_{y_{j}-\Delta_{1}}^{y_{j}} n_{1}(x, y) d x d y .
$$

Аналогичными выражениями можно описать и сигнал от подстилающей поверхности. Следовательно, мы можем записать:

$$
\begin{aligned}
& H_{1}: \xi(i, j)=n_{1}(i, j)+\dot{I}_{1}(i, j)=n_{1 i j}+\dot{I}_{1 i j}, \\
& H_{0}: \xi(i, j)=n_{0}(i, j)+\dot{I}_{0}(i, j)=n_{0 i j}+\dot{I}_{0 i j}
\end{aligned}
$$

где $i=1 \ldots m ; j=1 \ldots l ; m \times l-$ размеры матрицы ИК-приемника.

В рассматриваемом случае распределение внутренних шумов приемника является, как правило, нормальным. В соответствии с [8] плотность вероятности шума можно записать как

$$
\omega_{k \times m}\left(\ldots, n_{i j}, \ldots\right)=\prod_{i=1}^{l} \prod_{j=1}^{m} \frac{1}{\sqrt{2 \pi \bar{\sigma}_{\mathrm{m}}^{2}}} e^{-\frac{n_{i j}^{2}}{2 \bar{\sigma}_{\mathrm{I}}^{2}}}
$$

где $\bar{\sigma}_{ш}^{2}-$ средний квадрат (дисперсия) флуктуаций шума; $n_{i j}^{2}-$ квадрат значения шумов на выходе $i j$-го элемента приемника за время отсчета. 
Поэтому многомерные условные плотности распределения изображений на выходе приемника, соответствующие гипотезам $H_{1}$ и $H_{0}$ в предположении о статистической независимости значений сигналов и помех в элементах матрицы приемников, будут иметь вид

$$
\begin{aligned}
& \omega_{k \times m}\left(\ldots, \xi_{i j}, \ldots / H_{1}\right)=\prod_{i=1}^{l} \prod_{j=1}^{m} \frac{1}{\sqrt{2 \pi \bar{\sigma}_{\mathrm{II}}^{2}}} e^{-\frac{\left(\xi_{i j}-\dot{I}_{1 i j}-n_{1 i j}\right)^{2}}{2 \bar{\sigma}_{\mathrm{II}}^{2}}} ; \\
& \omega_{k \times m}\left(\ldots, \xi_{i j}, \ldots / H_{0}\right)=\prod_{i=1}^{l} \prod_{j=1}^{m} \frac{1}{\sqrt{2 \pi \bar{\sigma}_{\mathrm{II}}^{2}}} e^{-\frac{\left(\xi_{i j}-\dot{I}_{0 i j}-n_{0 i j}\right)^{2}}{2 \bar{\sigma}_{\mathrm{II}}^{2}}},
\end{aligned}
$$

где $\dot{I}_{1 i j}$ и $\dot{I}_{0 i j}-$ значения токов в элементе матрицы приемника от замаскированного объекта и ПП соответственно.

Применительно к задаче различения сигналов в данной ситуации наиболее приемлем критерий идеального наблюдателя [9]. Определим алгоритм принятия решения:

$$
\begin{aligned}
& \frac{\omega_{k \times m}\left(\ldots, \xi_{i j}, \ldots / H_{1}\right)}{\omega_{k \times m}\left(\ldots, \xi_{i j}, \ldots / H_{0}\right)}=\frac{P_{p r}\left(H_{1}\right)}{P_{p r}\left(H_{0}\right)} \prod_{i=1}^{l} \prod_{j=1}^{m} e^{\frac{\left(\xi_{i j}-\dot{I}_{0 i j}-n_{0 i j}\right)^{2}-\left(\xi_{i j}-\dot{I}_{1 i j}-n_{1 i j}\right)^{2}}{2 \bar{\sigma}_{\amalg}^{2}}}= \\
& =\frac{P_{p r}\left(H_{1}\right)}{P_{p r}\left(H_{0}\right)} e^{\frac{1}{2 \bar{\sigma}_{\amalg}^{2} \sum_{i=1}^{l} \sum_{j=1}^{m}\left(\xi_{i j}-\dot{I}_{0 i j}-n_{0 i j}\right)^{2}-\left(\xi_{i j}-\dot{I}_{1 i j}-n_{1 i j}\right)^{2}}>1,}
\end{aligned}
$$

где $P_{p r}\left(H_{1}\right)$ и $P_{p r}\left(H_{0}\right)$ - априорные вероятности.

Преобразуя выражения и логарифмируя обе части, получим

$$
\left.\sum_{i=1}^{l} \sum_{j=1}^{m} \xi_{i j}\left(\Delta \dot{I}_{i j}+\Delta n_{i j}\right)\right\rangle \bar{\sigma}_{u}^{2} \ln \frac{P_{p r}\left(H_{0}\right)}{P_{p r}\left(H_{1}\right)}+\sum_{i=1}^{l} \sum_{j=1}^{m} \frac{\left(\dot{I}_{1 i j}+n_{1 i j}\right)^{2}-\left(\dot{I}_{0 i j}+n_{0 i j}\right)^{2}}{2},
$$

где $\Delta \dot{I}_{i j}=\dot{I}_{1 i j}-\dot{I}_{0 i j}, \Delta n_{i, j}=n_{1 i, j}-n_{0 i j}$.

В соответствии с [9]

$$
\bar{E}_{1}(\Sigma)=\sum_{i=1}^{l} \sum_{j=1}^{m}\left(\dot{I}_{1 i j}+n_{1 i j}\right)^{2} ; \bar{E}_{0}(\Sigma)=\sum_{i=1}^{l} \sum_{j=1}^{m}\left(\dot{I}_{0 i j}+n_{0 i j}\right)^{2} .
$$

Учитывая, что априорные вероятности получения сигналов от истинной и ложной целей равны, алгоритм принятия решения примет следующий вид:

$$
q_{1}=\sum_{i=1}^{l} \sum_{j=1}^{m} \xi_{i j}\left(\Delta \dot{I}_{i j}+\Delta n_{i j}\right) \frac{H_{1} \geq}{H_{0}<}\left|\frac{\bar{E}_{1}(\Sigma)-\bar{E}_{0}(\Sigma)}{2}\right|=h .
$$

Структурная схема оптимального приемника, реализующего алгоритм (3.36), представлена на рис. 3.

Проведем анализ алгоритма, изображенного на рис. 3. Как видно из выражения (9), величина $q_{1}$ представляет собой сумму большого числа случайных величин и в соответствии с центральной предельной теоремой ее закон распределения может быть признан нормальным. Поэтому для определения вероятности суммарной ошибки $P_{c}\left(q_{1}\right)$ достаточно найти математи- 


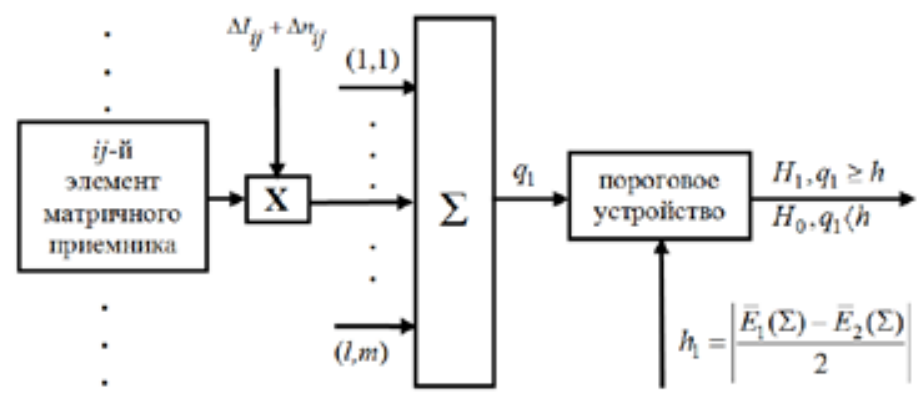

Рис. 3. Структурная схема оптимального приемника при различении двух изображений

Fig. 3. Optimal receiver structural schematics with the differences of two images

ческое ожидание и дисперсии плотности вероятности распределения $q_{1}$ при условии справедливости гипотез $H_{1}$ и $H_{0}$.

Если справедлива гипотеза $H_{1}$, то $\xi_{i j}=\dot{I}_{1 i j}+n_{1 i j}$, и математическое ожидание $\left\langle q_{1}\left(\dot{I}_{1}\right)\right\rangle$, и дисперсия $\sigma_{q_{1}\left(I_{1}\right)}^{2}$ будут определяться следующими выражениями:

$$
\begin{aligned}
& \left\langle q_{1}\left(\dot{I}_{1}\right)\right\rangle=\sum_{i=1}^{l} \sum_{j=1}^{m}\left(\dot{I}_{1 j}+n_{1 j}\right)\left(\Delta \dot{I}_{i j}+\Delta n_{i j}\right) ; \\
& \sigma_{q_{1}\left(\dot{I}_{1}\right)}^{2}=\sum_{i=1}^{l} \sum_{j=1}^{m}\left(\xi_{i j}-\left\langle q_{1}\left(\dot{I}_{1}\right)\right\rangle\right)^{2}=\sum_{i=1}^{l} \sum_{j=1}^{m}\left[\left(\dot{I}_{1 i j}+n_{1 i j}\right)\left(1-\left(\Delta \dot{I}_{i j}+\Delta n_{i j}\right)\right]^{2} .\right.
\end{aligned}
$$

Аналогично для гипотезы $H_{0}$ :

$$
\begin{aligned}
& \left\langle q_{1}\left(\dot{I}_{0}\right)\right\rangle=\sum_{i=1}^{l} \sum_{j=1}^{m}\left(\dot{I}_{0 i j}+n_{0 i j}\right)\left(\Delta \dot{I}_{i j}+\Delta n_{i j}\right) ; \\
& \sigma_{q_{1}\left(\dot{I}_{0}\right)}^{2}=\sum_{i=1}^{l} \sum_{j=1}^{m}\left(\xi_{i j}-\left\langle q_{1}\left(\dot{I}_{0}\right)\right\rangle\right)^{2}=\sum_{i=1}^{l} \sum_{j=1}^{m}\left[\left(\dot{I}_{0 i j}+n_{0 i j}\right)\left(1-\left(\Delta \dot{I}_{i j}+\Delta n_{i j}\right)\right]^{2} .\right.
\end{aligned}
$$

Тогда плотности вероятности распределения величины $q_{1}$ будут описываться выражениями:

$$
\begin{aligned}
& W_{\Sigma}\left(q_{1} / H_{1}\right)=\frac{1}{\sqrt{2 \pi} \sigma_{q_{1}\left(\dot{I}_{1}\right)}} e^{-\frac{\left(q_{1}-\left\langle q_{1}\left(\dot{I}_{1}\right)\right\rangle\right)^{2}}{2 \sigma_{q_{1}\left(\dot{I}_{1}\right)}^{2}}} ; \\
& W_{\Sigma}\left(q_{1} / H_{0}\right)=\frac{1}{\sqrt{2 \pi} \sigma_{q_{1}\left(\dot{I}_{0}\right)}} e^{-\frac{\left(q_{1}-\left\langle q_{1}\left(\dot{I}_{0}\right)\right\rangle\right)^{2}}{2 \sigma_{q_{1}\left(\dot{I}_{0}\right)}^{2}}} .
\end{aligned}
$$

Вероятность суммарной ошибки с учетом выбранного критерия и выражений (24) можно определить с помощью выражения

$$
\begin{aligned}
& P_{\mathrm{c}}\left(q_{1}\right)=P_{p r}\left(H_{1}\right) P\left(H_{0}^{*} / H_{1}\right)+P_{p r}\left(H_{0}\right) P\left(H_{1}^{*} / H_{0}\right)= \\
& =\frac{1}{2}\left(P\left(H_{0}^{*} / H_{1}\right)+P\left(H_{1}^{*} / H_{0}\right)\right)=\frac{1}{2}\left\{\int_{-\infty}^{h} W_{\Sigma}\left(q_{1} / H_{2}\right) d q_{1}+\int_{h}^{\infty} W_{\Sigma}\left(q_{1} / H_{0}\right) d q_{1}\right\},
\end{aligned}
$$


где $P_{p r}\left(H_{1}\right)=P_{p r}\left(H_{0}\right)=0,5$ - априорные вероятности появления в поле зрения замаскированного объекта и лишь ПП; $P\left(H_{0}^{*} / H_{1}\right)$ - вероятность принятия замаскированного объекта за ПП; $P\left(H_{1}^{*} / H_{0}\right)$ - вероятность принятия ПП за замаскированный объект.

Вероятности перепутывания будут определяться выражением

$$
P_{\text {пер }}^{(1)}=\int_{h}^{\infty} W_{\Sigma}\left(q_{1} / H_{0}\right) d q_{1} ; \quad P_{\text {пер }}^{(2)}=\int_{-\infty}^{h} W_{\Sigma}\left(q_{1} / H_{1}\right) d q_{1} .
$$

Полученные таким образом вероятности перепутывания позволяют оценить эффективность средства маскировки и кроме этого, что весьма важно, обеспечивают построение модели процесса поиска замаскированного объекта в условиях преднамеренных помех.

Для исследования зависимости вероятностей $P_{c}, P_{\text {пер }}^{(1)}$ и $P_{\text {пер }}^{(2)}$ от значений сигналов и шумов было проведено математическое моделирование на ПЭВМ в среде Matcad. Пример распределения интенсивностей по поверхностям изображений подстилающей поверхности и замаскированного объекта приведен на рис. 4.

В качестве тестового изображения задано изображение квадрата размерностью 10х10 элементов с неоднородной интенсивностью излучения (среднее значение интенсивности 55-60). Каждый элемент изображения соответствует элементу матрицы фотоприемника с соответствующими этому элементу значениями токов, возникающих в матрице приемника. В центре изображения задавался тестовый замаскированный объект в виде квадрата размерностью 4х4 элемента, имеющий увеличенную интенсивность.

Для оценки вероятности суммарной ошибки от степени подобия изображений в изображении замаскированного объекта изменялись значения элементов ( $K$ элементов имели интенсивность большую, чем в изображении подстилающей поверхности, на 10-90 \%), при этом уровень шумов был одинаковым.

На рис. 5 представлены графики зависимости вероятности суммарной ошибки от степени подобия изображений.

Из анализа полученных зависимостей можно сделать следующие выводы. При равных значениях шумов (в нашем случае $n_{1}=n_{2}=10$ ) в изображениях замаскированного объекта и

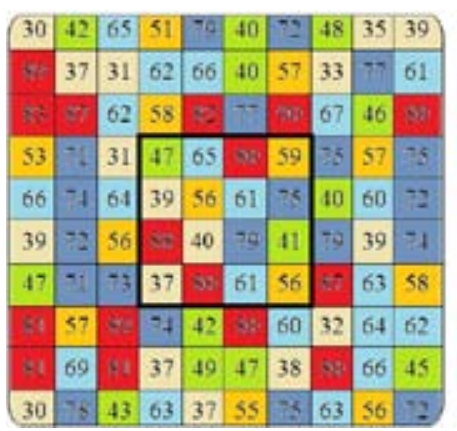

$H_{1}$

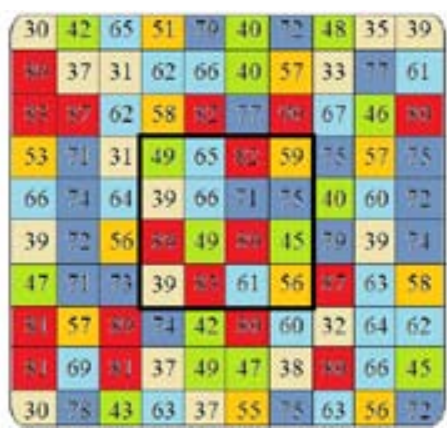

$H_{0}$

Рис. 4. Пример распределения интенсивностей на изображениях замаскированного объекта и подстилающей поверхности

Fig. 4. Example of intensity pattern on camouflaged object' and underlying surface images 


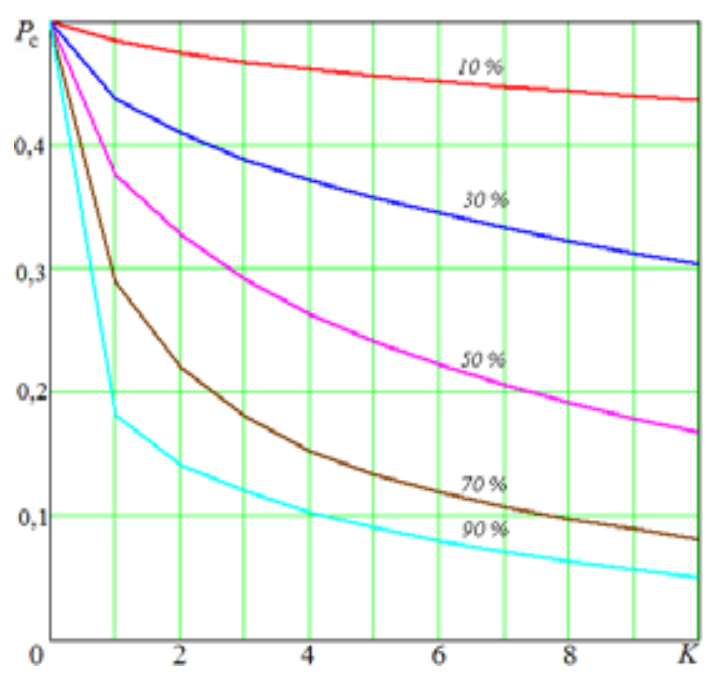

Рис. 5. Зависимости $P_{\mathrm{c}}$ от степени подобия изображений

Fig. 5. $P_{\mathrm{c}}$ dependencies on images degree

подстилающей поверхности различие в уровнях сигнала в 5-10 \% не оказывает существенного влияния на вероятностные показатели различения. Для достижения максимальных значений вероятности суммарной ошибки и перепутывания при прикрытии объектов маскировочными средствами будет эффективным применение активных маскирующих помех, подобранных по мощности, при этом уровень необходимой мощности помехи не слишком отличается (на единицы процентов) от уровня мощности сигнала.

Таким образом, с использованием метода интегрирования по источнику получено амплитудное распределение излучения, исходящего от прикрываемого объекта, по внешней поверхности маскирующего средства. Путем его вложения в суммарное распределение общей площади, ограниченной полем зрения разведывательного ИК-приемника, можно оценить составное распределение в предметной плоскости с дальнейшим переносом изображения из рассматриваемой плоскости в фокальную плоскость системы разведки. Это позволило разработать методический подход к оценке эффективности маскирующих средств, позволяющий достаточно строго учитывать распределения интенсивностей излучения по поверхностям объекта и подстилающей поверхности.

\section{Список литературы}

[1] Козирацкий Ю.Л., Будников С.А., Иванцов А.В. и др. Модели информационного конфликта средств поиска и обнаружения. Монография. Москва: Радиотехника, 2013. 232 с. [Koziratsky Yu.L., Budnikov S.A., Ivantsov A.V. et al Models of informational conflict of search and detection. Monography. Moscow, Radiotechnics, 2013. 232 p. (in Russian)]

[2] Козирацкий Ю.Л., Донцов А.А., Иванцов А.В. и др. Модели пространственного и частотного поиска. Монография. / Под ред. Ю.Л. Козирацкого. Москва: Радиотехника, 2014. 344 c. [Koziratsky Yu.L., Dontsov A.A., Ivantsov A.V. et ai Models of dimensional and frequency search. Monography. Moscow, Radiotechnics, 2014. 344 p. (in Russian)] 
[3] Козирацкий Ю.Л., Алабовский А.В. Особенности различения оптических сигналов, подчиняющихся пуассоновской статистике. Москва: Радиотехника, 1989. № 1. [Koziratsky Yu.L., Alabovskii A.V. Particularity of optical signal dependencies based on Poisson statistics. Moscow, Radiotechnics. 1989. №1 (in Russian)]

[4] Козирацкий Ю.Л., Тимохин В.Н., Козирацкий А.Ю. Оценочно-компенсационный алгоритм выделения кратковременно существующих изображений матричными приемниками. Москва: Радиотехника, 2004. № 5. 96-99 с. [Koziratsky Yu.L., Timokhin V.N., Koziratsky A.Yu. Evaluation and estimation algorithm for allocation of shot existent images by detector arrays. Moscow, Radiotechnics, 2004. №5. 96-99 p. (in Russian)]

[5] Козирацкий, Ю.Л., Иванцов А.В., Козирацкий А.Ю. Оценочно-компенсационный алгоритм различения кратковременно существующих изображений приемниками, работающими в режиме счета электронов. Москва: Радиотехника, 2004. № 5. 43-48 с. [Koziratsky Yu.L., Ivantsov A.V., Koziratsky A.Yu. Evaluation and estimation algorithm for distinction of short existent images by receiver that works in electron counting regime. Moscow, Radiotechnics, 2004. №5. 43-48 p. (in Russian)]

[6] Козирацкий, Ю.Л., Иванцов А.В. Алгоритм различения изображений, имеющих одинаковые геометрические размеры. Москва: Радиотехника, 2005. № 7. 82-84 c. [Koziratsky Yu.L., Ivantsov A.V. Algorithm for distinction of images that have similar geometric measurements. Moscow, Radiotechnics, 2005. №7. 82-84 p. (in Russian)]

[7] Бакут, П.А., Мандросов В.И., Матвеев Н.Н., Устинов Н.Д. Теория когерентныхх изображений. Москва: Радио и связь, 1987. 264 с. [Bakut P.A., Mandrosov V.I., Matveev N.N., Ustinov N.D. Coherent images theory. Moscow, Radio and connection, 1987. 264 p. (in Russian)]

[8] Красильников Н.Н. Теория передачи и восприятия изображений. Москва: Радио и связь, 1986. 247 c. [Krasilnikov N.N., Theory of transmission and reception of images. Moscow, Radio and connection, 1986. 247 p. (in Russian)]

[9] Сосулин Ю.Г. Теория обнаружения и оценивания статистических сигналов. Москва: Сов. радио, 1978. 320 с. [Sosulin Yu.G. Theory of detection and estimation of statistics signals. Moscow, Soviet Radio, 1978. 320 p. (in Russian)]

[10] Курикша А.А. Квантовая оптика и оптическая локачия. Москва: Сов. радио, 1973. 183 c. [Kuriksha A.A. Quantum optics and optical location. Moscow, Soviet Radio, 1973. 183 p. (in Russian)]

[11] Шереметьев А.Г. Статистическая теория лазерной связи. Москва: Связь, 1971. 264 с. [Sheremetev A.G. Statistics theory for laser connection. Moscow, Signal, 1971. 264 p.] 